\title{
ANÁLISE DA ADAPTAÇÃO DOS ALUNOS INGRESSANTES EM 2020 AO REGIME DE ENSINO REMOTO NO CURSO DE ENGENHARIA DE MINAS DA UFC EM CRATEÚS
}

DOI: 10.37702/2175-957X.COBENGE.2021.3451

Francisco Wylhan Pereira dos Santos - pereirawylhan@gmail.com

Universidade Federal do Ceará UFC

Rua Tabelião Francisco Antero 1010

63702-880 - Crateús - CE

Andreza Rafaela Morais Pereira - andrezamorais00@gmail.com

UFC

Rua Francisco Zeca Silva 100

58410-817 - Campina Grande - PB

RAFAEL CHAGAS SILVA - rafaelchagas.silva@gmail.com

Universidade Federal do Ceará

Rua Jose Vieira De Lima 88

58417-393 - Campina Grande - PB

Kennedy da Silva Ramos - kennedyramos@crateus.com.br

Universidade Federal do Ceará

Rua: Gentil Barreiras 150

63702-260 - Crateús - CE

Tiany Guedes Cota - tianycota@crateus.ufc.br

Universidade Federal do Ceará

Rua Clovis Bevilaqua 1188

63700-155 - Crateús - CE

Resumo: Ao iniciar um curso de graduação, os ingressantes se deparam com vários desafios relacionados a diferentes fatores, como dificuldades de adaptabilidade ao ambiente universitário e nos componentes curriculares do ciclo básico. Estas adversidades podem levar os alunos a perderem a motivação pelo ensino, o que resulta no aumento dos casos de evasão e retenção em disciplinas iniciais do curso de Engenharia de Minas da Universidade Federal do Ceará (UFC), 
campus de Crateús. Além disso, no ano de 2020, a pandemia do Covid-19 trouxe vários desafios para a educação, como o distanciamento social e a dificuldade de adaptação ao ensino remoto. Neste sentido, este trabalho teve como objetivo identificar e analisar as principais dificuldades de adaptação enfrentadas pelos ingressantes no primeiro período de 2020 do curso de Engenharia de Minas da UFC em Crateús. Para tanto, foi aplicado um questionário eletrônico com a finalidade de coletar informações referentes à percepção das aulas remotas. De forma complementar, foram realizadas análises do rendimento acadêmico, quantidade de discentes que realizaram a supressão e trancamento total de disciplinas. $O$ questionário contou com a participação de 26 alunos, cuja representatividade foi de $54,2 \%$ da turma. Observou-se que $50 \%$ desses alunos classificaram as aulas remotas como regulares ou ruins. Em relação ao rendimento acadêmico, 22,9\% da turma obtiveram aprovação em todas as disciplinas no semestre. Quanto a supressão de disciplinas, 21 alunos solicitaram a supressão de disciplinas e 6 realizaram o trancamento total. Assim, acredita-se que a universidade tem um papel protagonista na elaboração de ações de permanência, possibilitando não apenas uma melhor integração dos estudantes ao ambiente acadêmico, mas também motivação e condições para o aluno progredir no curso.

Palavras-chave: Adaptação universitária. Permanência universitária. Ensino Remoto. Engenharia de Minas. 


\section{ANÁLISE DA ADAPTAÇÃO DOS ALUNOS INGRESSANTES EM 2020 AO REGIME DE ENSINO REMOTO NO CURSO DE ENGENHARIA DE MINAS DA UFC EM CRATEÚS}

\section{INTRODUÇÃO}

$\mathrm{Na}$ chegada ao ensino superior, os estudantes se deparam com vários desafios, alguns deles variam de acordo com a região do país e a instituição de ensino. Esses desafios podem estar atrelados a dificuldades de adaptação ao ambiente acadêmico, dificuldades com os componentes curriculares do ciclo básico e a fatores financeiros. Essas adversidades podem fazer com que os alunos percam a motivação pelo ensino, o que contribui para uma maior evasão e retenção em disciplinas (PEREIRA, 2003; FEY et al., 2013; DE OLIVEIRA et al., 2019). Souza et al. (2019) realizaram um estudo do perfil do ingressante no curso de Engenharia de Minas da UFC em Crateús, no qual se observou, a partir dos relatos dos alunos, que os mesmos enfrentam desafios diários e críticos, como a diferença de rigor educacional, exigência de considerável nível de conhecimentos básicos, maior volume e rapidez na transmissão de conhecimento, além de fatores financeiros e emocionais.

Além disso, a chegada da pandemia de COVID-19, surgiram outros desafios para a educação, a exemplo do distanciamento social e a busca de suporte necessário para o oferecimento das aulas remotas. Assim, as problemáticas que já existiam se agravaram nesse período (DIAS \& PINTO; MIRANDA et al.; SOUZA, 2020). Nesta perspectiva, foi necessário buscar novas alternativas e ferramentas virtuais, para que fosse possível ambientar os alunos ingressantes na universidade, além de ajudar no seu desenvolvimento acadêmico.

Conforme Santos et al. (2020) as universidades necessitaram reinventar-se por meio do ensino remoto emergencial, para dar continuidade as atividades através de plataformas virtuais, seja com interações síncronas ou assíncronas. Essa modalidade exigiu do corpo docente e dos gestores educacionais uma rápida tomada de decisão para continuar o processo de ensino-aprendizagem dos estudantes, bem como adaptar-se à utilização das tecnologias digitais para um viés didático-pedagógico.

Neste contexto, o presente trabalho teve como principal objetivo identificar e analisar as principais dificuldades enfrentadas pelos ingressantes do primeiro período letivo de 2020 do curso de Engenharia de Minas da UFC em Crateús, no intuito de entender e auxiliar nas dificuldades, a fim de ajudá-los a superar as adversidades geradas pelo ensino remoto.

\section{METODOLOGIA}

Para a realização da pesquisa utilizou-se um formulário eletrônico, disponibilizado aos alunos ingressantes do primeiro semestre, em 2020, do curso de Engenharia de Minas da UFC, campus de Crateús. O formulário criado na plataforma "Google Forms", contou com perguntas sucintas com o objetivo de obter informações sobre a percepção dos alunos sobre as aulas remotas, classificação do aprendizado e principais dificuldades enfrentadas nesse período. As perguntas abordadas no questionário estão listadas a seguir.

1. Como você classifica as aulas remotas? 
2. Como você considera o seu nível de aprendizagem durante as aulas remotas?

3. Como eu percebo minha interação com os colegas do meu curso?

4. Como eu percebo minha interação com os professores do meu curso?

5. Quais as dificuldades na modalidade de ensino remoto?

Além disso, com os dados fornecidos pela coordenação do curso de Engenharia de Minas, realizou-se o levantamento do rendimento acadêmico dos alunos ingressantes no curso e o quantitativo de alunos que realizaram a supressão total ou parcial das disciplinas.

\section{RESULTADOS E DISCUSSÃO}

O questionário aplicado contou com a participação de 26 alunos do curso de Engenharia de Minas da UFC em Crateús, de um total de 48 alunos ativos ingressantes no primeiro período de 2020 , o que representa $54,2 \%$ dos alunos matriculados.

A Figura 1 mostra os resultados referentes à classificação das aulas remotas de acordo com a percepção dos alunos, sendo possível observar que $50 \%$ dos discentes classificaram as aulas remotas como regulares ou ruins. $O$ fato de metade dos estudantes não observarem as aulas remotas de forma positiva pode ser o reflexo de várias questões como a falta de preparação dos alunos para estudar utilizando plataformas de ensino, não possuir lugar adequado de estudo, ou a falta de preparação dos professores para lidar com os recursos tecnológicos (MIRANDA et al., 2020). Essas questões podem interferir na adaptação do aluno ao ambiente universitário, uma vez que as aulas on-line estão sendo o seu primeiro contato com a universidade. Ademais, esses problemas podem acarretar prejuízos futuros como baixo nível de aprendizagem e pouco interesse pelos estudos na modalidade remota, podendo assim, aumentar o número de evasão escolar.

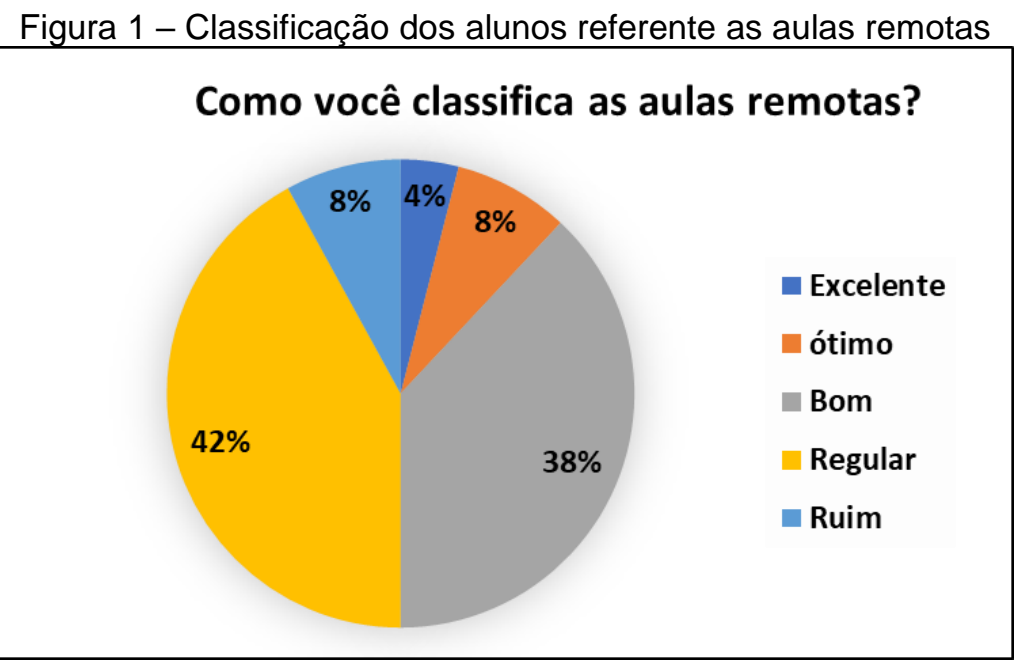

Fonte: Elaborado pelos autores (2021)

A Figura 2 mostra que $46 \%$ dos alunos consideram seu nível de aprendizagem durante as aulas remotas como regular ou ruim. Conforme observado na pergunta anterior, na qual $50 \%$ dos estudantes classificam as aulas remotas como ruins ou regulares, isso também reflete na percepção que os alunos têm dos seus níveis de aprendizado durante o período das aulas on-line. Essa percepção dos discentes pode 
ocorrer devido à urgente implementação do ensino remoto emergencial, com isso é possível que as limitações de suporte técnico, treinamento, tempo e planejamento tenham comprometido a qualidade do ensino, refletindo em possível consequências observadas pelas Instituições de Ensino Superior (HODGES et al.; GUSSO et al., 2020). Segundo Sanz et al. (2020) a primeira consequência a ser observada na substituição das aulas presenciais pelas aulas on-line é na aprendizagem dos alunos. Assim, é de extrema importância o envolvimento dos professores e o suporte da universidade no ensino remoto.

Figura 2 - Nível de aprendizado durante as aulas remotas

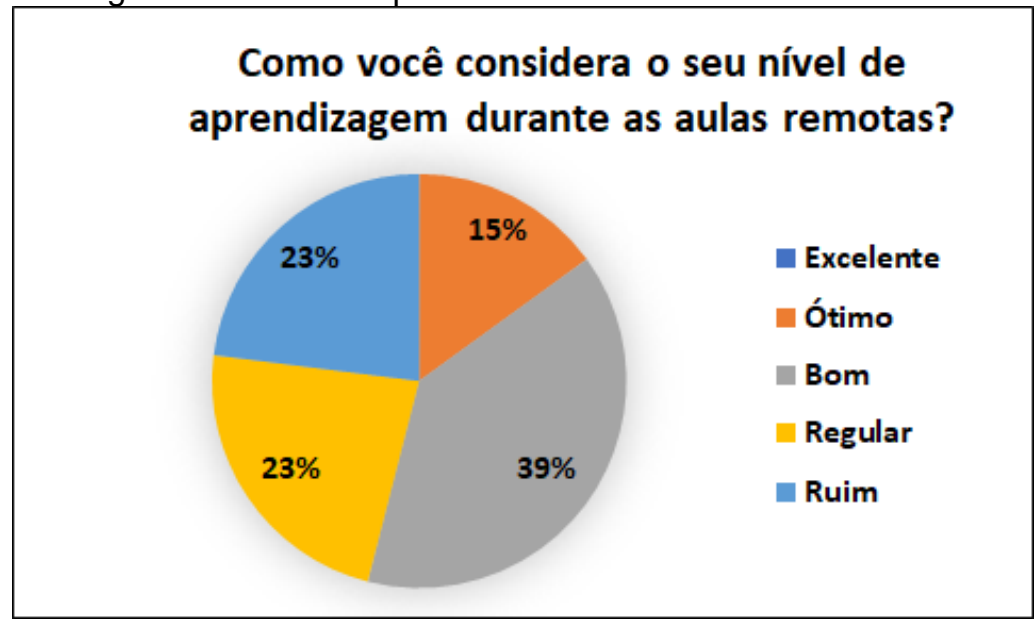

Fonte: Elaborado pelos autores (2021)

A Figura 3 apresenta que $46 \%$ dos alunos participantes da pesquisa consideram regular a interação com os professores do curso, enquanto que $38 \%$ consideram regular a interação com os colegas de curso. Esses resultados podem estar relacionados à dificuldade de comunicação nos ambientes virtuais, ou seja, o contato no ambiente virtual tende a ser menor do que no presencial. Segundo Wandscheer (2020), o fato dos alunos estarem inseridos em um mundo tecnológico poderia auxiliar nas atividades, aulas e provas on-line, mas o que não se tinha conhecimento era que para os alunos a interação presencial com os professores era fundamental.

Figura 3 - Interação com os professores e com os colegas do curso

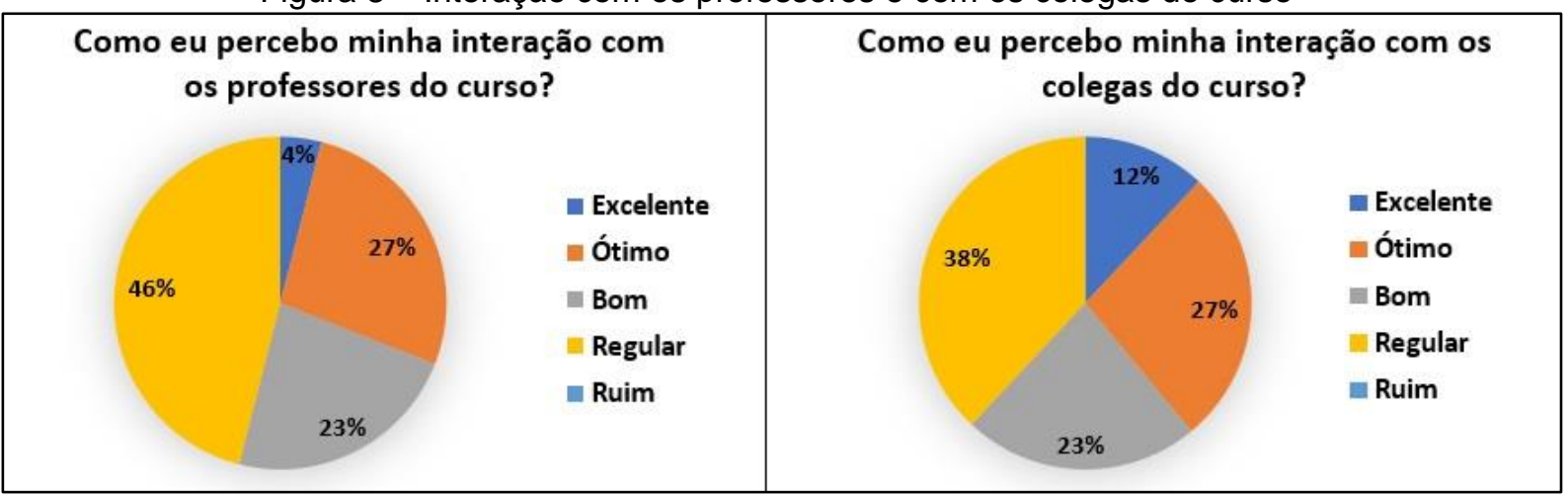

Fonte: Elaborado pelos autores (2021)

Ainda que o ensino remoto e a interação entre professores e alunos só foi possível devido às plataformas digitais, os computadores, os notebooks, os celulares, e à internet, é preciso destacar que, apesar de todos esses novos recursos 
técnológicos utilizados para manter o ensino de forma remota, não acredita-se que somente por meio dessas tecnologias a educação aconteça (WANDSCHEER, 2020). Segundo Levy (1996), a educação consiste da presença das pessoas por ela entrelaçadas. O ato de ensinar e aprender é um ato de amorosidade (FREIRE, 1996; WANDSCHEER, 2020).

A Figura 4 mostra os resultados obtidos referentes as principais dificuldades enfrentadas no ensino remoto, sendo possível observar que as predominantes adversidades apontadas pela maioria dos alunos foram o planejamento e a organização das atividades, e conciliar as atividades acadêmicas e pessoais. Uma vez que agora o ambiente de lazer e de estudos passaram a ser os mesmos, isso requer dos alunos maior responsabilidade. No caso dos ingressantes que estão conhecendo o ambiente acadêmico, essas dificuldades atrapalham ainda mais a adaptação.

Figura 4 - Principais dificuldades enfrentadas pelos alunos na modalidade remoto

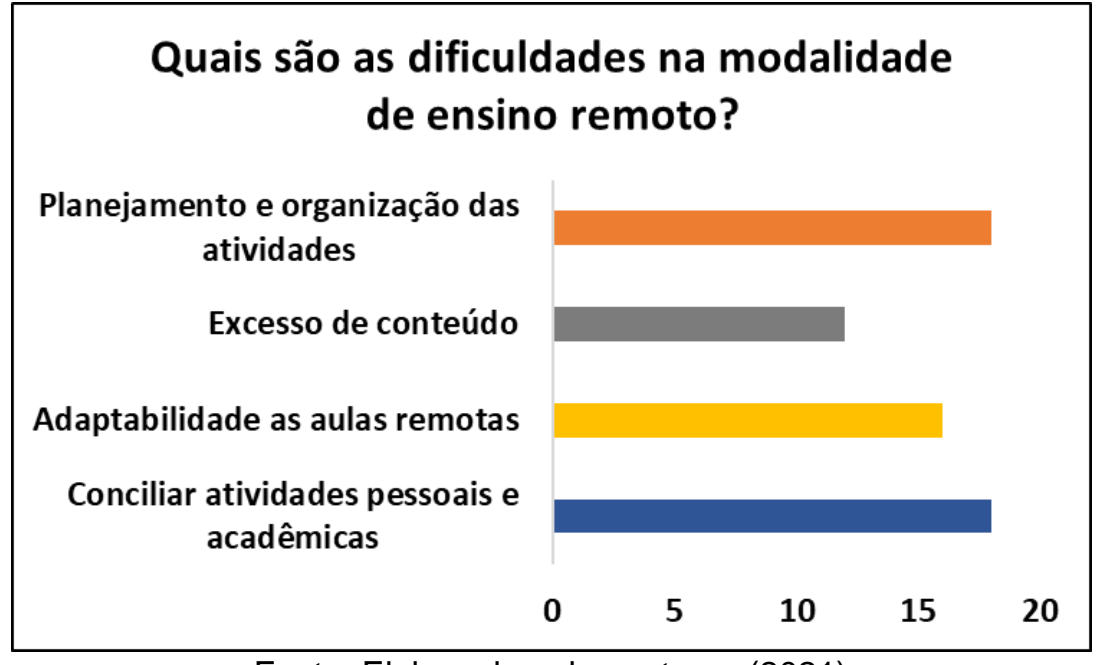

Fonte: Elaborado pelos autores (2021)

Estes resultados corroboram o estudo de Miranda et al. (2020), o qual descreve que os alunos têm relatado problemas como falta de motivação e de um lugar adequado para estudar, gerando dificuldades na compreensão dos assuntos, além de ausência de organização e planejamento de horários de estudos. O ensino vem sofrendo com as consequências, diante de todos os infortúnios ocasionados pela pandemia desde estudantes, pais, até professores nos mais variados níveis de ensino. No período da pandemia ocorreram várias mudanças as quais geram interferências nas famílias que passaram a conviver cotidianamente com vários conflitos, além de interferir na aprendizagem e perspectivas futuras da maioria dos discentes (MÉDICE ET AL., 2020; MIRANDA ET AL., 2020, DE SOUZA, 2020).

De acordo com os dados fornecidos pela coordenação do curso de Engenharia de Minas, realizou-se o levantamento do rendimento acadêmico dos alunos ingressantes no curso no ano de 2020. Atualmente, 48 alunos estão ativos no curso desde o primeiro semestre de 2020, dentre estes, 11 obtiveram sucesso em todas as disciplinas do primeiro semestre, representando aproximadamente $23 \%$ dos alunos ingressantes. Por outro lado, cerca de $77 \%$ dos alunos reprovaram em pelo menos uma disciplina, conforme mostrado na Figura 5. Essa alta taxa de reprovação ou insucesso dos discentes pode ser devido a problemas de saúde mental ou física decorrente do período de confinamento. Consoante Dias e Pinto (2020), 0 confinamento prolongado, a falta de contato com os colegas de classe, a falta de 
espaço em casa, além do medo de ser infectado podem afetar a saúde dos estudantes.

Figura 5 - Rendimento acadêmico dos alunos ingressantes

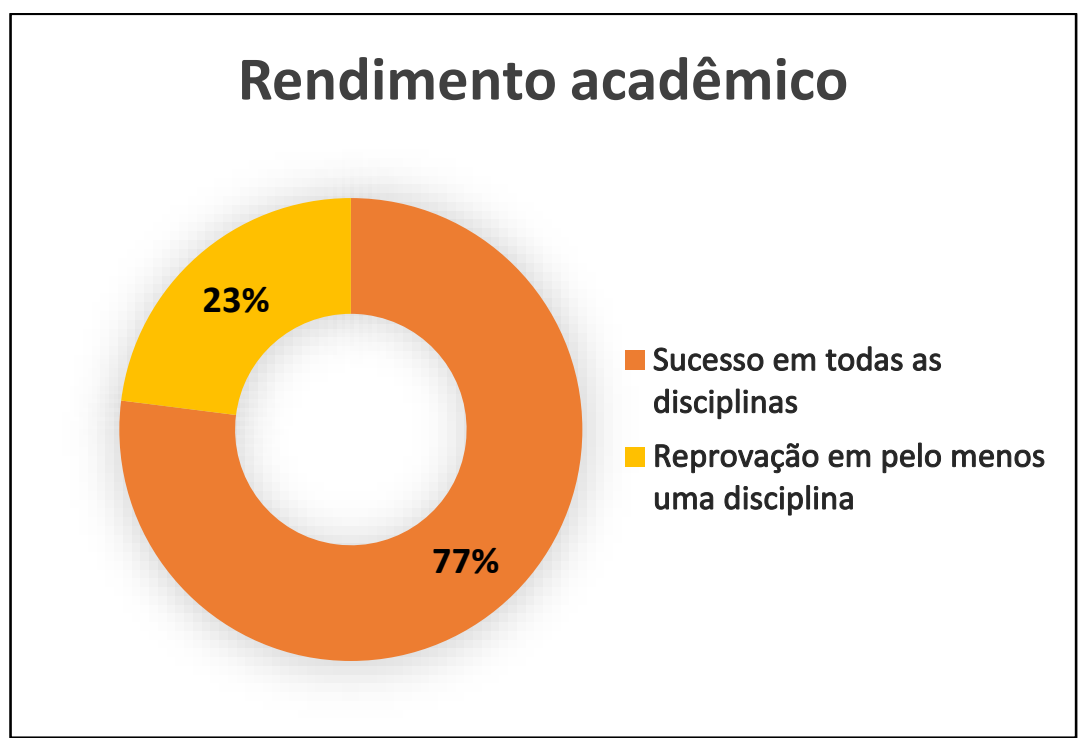

Fonte: Elaborado pelos autores (2021)

Com relação à supressão de disciplinas, registrou-se 27 solicitações, entre estes 6 alunos realizaram o trancamento total das disciplinas do semestre letivo. A Figura 6 mostra que $70 \%$ dos alunos não conseguiram acompanhar as aulas no formato remoto, o que resultou na supressão de disciplinas de forma parcial ou total. Este percentual elevado pode estar relacionado à incompatibilidade desses alunos com as aulas no formato on-line decorrente da falta de acesso à internet a falta de um equipamento eletrônico adequado para assistir as aulas ou da falta de preparação dos estudantes para a utilização das plataformas digitais, além do impacto psicológico negativo da pandemia nos estudantes. (MAIA \& DIAS; DIAS \& PINTO, 2020).

Figura 6-Quantidade de alunos que conseguiram acompanhar as aulas remotas

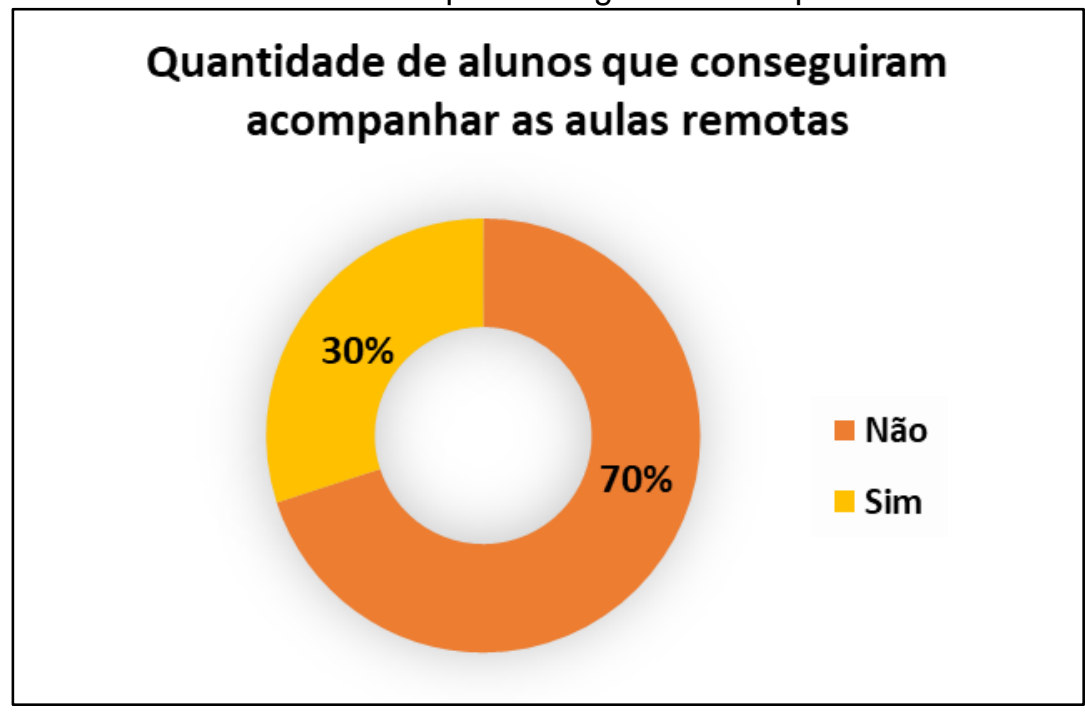

Fonte: Elaborado pelos autores (2021) 


\section{CONSIDERAÇÕES FINAIS}

A partir dos resultados apresentados neste trabalho foi possível observar os aspectos que influenciaram na permanência e na adaptação dos discentes ingressantes do primeiro semestre de 2020 ao curso de engenharia de minas da UFC em Crateús. Por meio dos dados obtidos, foi possível concluir que as aulas remotas afetaram à adaptação ao ambiente universitário e à interação com os docentes e colegas de turma, além da aprendizagem de grande parte dos alunos ingressantes nas componentes curriculares do ciclo básico, levando-os a reprovação ou supressão de um ou mais disciplinas do curso, acarretando em perdas aos estudantes e a Instituição de ensino. Esses prejuízos são decorrentes principalmente da urgência da implementação do ensino remoto emergencial devido à pandemia de Covid-19, esta metodologia de ensino expôs as desigualdades já existentes, que são, em parte, niveladas no ambiente presencial. Portanto, a universidade terá um papel protagonista para que se elabore ações de permanência para esses alunos, pois assim possibilitará não apenas uma melhor integração desses estudantes no ambiente acadêmico, como também, poderá influenciar positivamente na motivação e dar condições para que esses alunos possam progredir no curso.

\section{Agradecimentos}

Os autores agradecem a Universidade Federal do Ceará pela concessão da bolsa do Programa de Acolhimento e Incentivo a Permanência (PAIP).

\section{REFERÊNCIAS}

DE OLIVEIRA, C. H. M.; SANTOS, F. R. T.; LEITINHO, J. L.; FARIAS, L. G. A. T. Busca dos fatores associados à evasão: um estudo de caso no Campus Universitário da UFC em Crateús. Revista Internacional de Educação Superior. Campinas, v.5 p. 1-23, 2019.

DE SOUZA, E. P. Educação em tempos de pandemia: desafios e possibilidades. Cadernos de Ciências Sociais Aplicadas, ano XVII, v. 17, n. 30, p. 110-118, 2020.

DIAS, E.; PINTO, F. C. F. A Educação e a Covid-19. Revista Ensaio: Avaliação e Políticas Públicas em Educação, Rio de Janeiro, v.28, n.108, p. 545-554, 2020.

FEY, A. F.; LUCENA, K. C.; FOGAÇA, V. N. S. Evasão no ensino superior: Uma pesquisa numa IES do ensino privado. Revista de Humanidades, Tecnologia e Cultura, v. 1, n. 1, 2013.

FREIRE, P. Pedagogia da autonomia: Saberes necessários à prática educativa. São Paulo: Editora Paz e Terra, 1996.

GUSSO, H. L. Ensino superior em tempos de pandemia: Diretrizes à gestão universitária. Educação \& Sociedade, Campinas, v. 41, p. 1-27, 2020. 
HODGES, C.; MOORE, S.; LOCKEE, B.; TRUST, T.; BOND, A. The difference between emergency remote teaching and online learning. Disponível em: https://er.educause.edu/articles/2020/3/the-difference-between-emergency-remoteteaching-and-online-learning. Acesso em: 31 mar. 2021.

LÉVY, P. O que é virtual?. São Paulo: Editora 34, 1996.

MAIA, B. R.; DIAS, P. C. Ansiedade, depressão e estresse em estudantes universitários: o impacto da COVID-19. Estudos de Psicologia (Campinas), Campinas, v. 37, p. 1-8, 2020.

MÉDICI, M. S.; TATTO, E. R.; LEÃO, M. F. Percepções de estudantes do Ensino Médio das redes pública e privada sobre atividades remotas ofertadas em tempos de pandemia do coronavírus. Revista Thema, v. 18, n. Especial COVID-19, p. 136-155, 2020.

MIRANDA, Kacia Kyssy Câmara de Oliveira et al. Aulas remotas em tempo de pandemia: desafios e percepções de professores e alunos. In: VII Congresso Nacional de Educação, 2020, Maceió. Anais. Maceió. Disponível em: https://editorarealize.com.br/artigo/visualizar/68086. Acesso em: 02 abr. de 2021.

PEREIRA, F. C. B. Determinantes da evasão de alunos e os custos ocultos para as Instituições de Ensino Superior: Uma aplicação na universidade do extremo Sul Catarinense. Doutorado - Universidade Federal de Santa Catarina, 2003.

SANTOS, G. M. T.; REIS, J. P. C.; MÉRIDA, E. C.; RANGEL, E. L. F.; FRICH, A.A. Educação superior: reflexões a partir do advento da pandemia da COVID-19. Boletim de Conjuntura (BOCA), v. 4, n. 10, p. 108-114, 2020.

\section{SANZ, I.; SAINZ, J.; CAPILLA, A. Efeitos da crise do Covid-19 na educação.} Disponível em:

https://observatoriodeeducacao.institutounibanco.org.br/cedoc/detalhe/tf-oei-efeitosda-crise-do-covid-19-na-educacao,a2fb7f17-c150-40f0-9433-71095dc9ff38. Acesso em 01 abr. de 2021.

SOUZA, D. M.; COTA, T. G.; LIMA, S. V.; RAMOS, K. S.; Estudo do perfil do ingressante do curso de Engenharia de Minas do campus da Universidade Federal do Ceará em Crateús. In: XLVII Congresso Brasileiro de Educação em Engenharia, 2019, Fortaleza. Anais. Fortaleza. Disponível em: http://www.abenge.org.br/sis_artigo_doi.php?e=COBENGE\&a=19\&c=2476. Acesso em 20 de jan.2020.

WANDSCHEER, K. T. Ensino remoto: Um caminhar de possibilidades educativas. In: PALÚ, J.; SCHÜTZ, J. A.; MAYER, L. (Org.). Educação em tempos de pandemia. 1 ed. Cruz Alta: Editora llustração, 2020. p. 235-246. 


\title{
ANALYSIS OF THE ADAPTATION OF INGRESSING STUDENTS IN 2020 TO THE REMOTE TEACHING IN THE MINING ENGINEERING COURSE OF UFC IN CRATEÚS
}

\begin{abstract}
When starting an undergraduate course, the newcomers come across various challenges related to different factors, such as difficulties in adapting to the university environment and the curricular components of the basic cycle. These adversities can lead students to lose motivation for learning, which results in an increase in cases of dropout and retention in initial subjects of the Mining Engineering course at the Federal University of Ceará (UFC), campus of Crateús. Moreover, the Covid-19 pandemic has brought with it several challenges for education, such as social distancing and the difficulty of adapting to remote education. In this sense, this work aimed to identify and analyze the main difficulties faced by those entering the first period of 2020 in the Mining Engineering course at UFC in Crateús. To this end, an electronic questionnaire was applied in order to collect information regarding the perception of remote classes. In a complementary way, it was carried out analyses of academic performance, the number of students who made the suppression, and total cancellation of subjects. The questionnaire had the participation of 26 students, whose representation was $54.2 \%$ of the class. It was observed that $50 \%$ of the students classified the remote classes as regular or bad. Regarding academic performance, $22.9 \%$ obtained approval in all subjects in the semester. Regarding the suppression of disciplines, 21 students requested the suppression of disciplines and 6 performed the total shutdown. In this sense, it is believed that the university has a leading role in the development of permanence actions, enabling not only a better integration of students to the academic environment, but also motivation and conditions for the student to progress in the undergraduate course.
\end{abstract}

Keywords: University adaptation. University permanence. Remote Teaching. Mining Engineering. 